\title{
Modulation of Gut Microbiota by Lonicera caerulea L. Berry Polyphenols in a Mouse Model of Fatty Liver Induced by High Fat Diet
}

\author{
Shusong $\mathrm{Wu}^{1,2}{ }^{1,}$ Ruizhi Hu ${ }^{1}$, Hironobu Nakano ${ }^{3}, \mathrm{Keyu}_{\mathrm{Chen}}{ }^{3}, \mathrm{Ming} \mathrm{Liu}^{1}{ }^{1} \mathrm{Xi} \mathrm{He}^{1}$, \\ Hongfu Zhang ${ }^{2}$, Jianhua He ${ }^{1, *}$ (D) and De-Xing Hou ${ }^{1,3, *}$ \\ 1 Core Research Program 1515, Hunan Collaborative Innovation Center for Utilization of Botanical Functional \\ Ingredients, Hunan Agricultural University, Changsha 410128, China; wush688@126.com (S.W.); \\ rzhi479@163.com (R.H.); liuming@hongkebio.cn (M.L.); hexi111@126.com (X.H.) \\ 2 The State Key Laboratory of Animal Nutrition, China Agricultural University, Beijing 100193, China; \\ zhanghongfu@caas.cn \\ 3 The United Graduate School of Agricultural Sciences, Kagoshima University, Kagoshima 890-0065, Japan; \\ k8108910@kadai.jp (H.N.); k4164345@kadai.jp (K.C.) \\ * Correspondence: jianhuahy@hunau.net (J.H.); hou@chem.agri.kagoshima-u.ac.jp (D.-X.H.); \\ Tel.: +86-731-846-18176 (J.H.); +81-992858649 (D.-X.H.)
}

Received: 30 October 2018; Accepted: 27 November 2018; Published: 5 December 2018

check for updates

\begin{abstract}
Polyphenols from the Lonicera caerulea L. berry have shown protective effects on experimental non-alcoholic fatty liver disease (NAFLD) in our previous studies. As endotoxins from gut bacteria are considered to be the major trigger of inflammation in NAFLD, this study aims to clarify the regulatory effects of L. caerulea L. berry polyphenols (LCBP) on gut microbiota in a high fat diet (HFD)-induced mouse model. C57BL/6N mice were fed with a normal diet, HFD, or HFD containing $0.5-1 \%$ of LCBP for 45 days. The results revealed that supplementation with LCBP decreased significantly the levels of IL-2, IL-6, MCP-1, and TNF- $\alpha$ in serum, as well as endotoxin levels in both serum and liver in HFD-fed mice. Fecal microbiota characterization by high throughput $16 \mathrm{~S}$ rRNA gene sequencing revealed that a HFD increased the Firmicutes/Bacteroidetes ratio, and LCBP reduced this ratio by increasing the relative abundance of Bacteroides, Parabacteroides, and another two undefined bacterial genera belonging to the order of Bacteroidales and family of Rikenellaceae, and also by decreasing the relative abundance of six bacterial genera belonging to the phylum Firmicutes, including Staphylococcus, Lactobacillus, Ruminococcus, and Oscillospira. These data demonstrated that LCBP potentially attenuated inflammation in NAFLD through modulation of gut microbiota, especially the ratio of Firmicutes to Bacteroidetes.
\end{abstract}

Keywords: polyphenols; Lonicera caerulea L.; fatty liver disease; inflammation; gut microbiota

\section{Introduction}

Phytochemicals present in functional foods, such as cool climate berries, offer a great hope as an alternative therapy for chronic disorders [1]. Lonicera caerulea L., also known as blue honeysuckle, honeyberry or haskap, is a member of the Caprifoliaceae family that grows naturally in cool temperate regions in the Northern Hemisphere, such as Siberia in Russia, Hokkaido in Japan, and northern China, and has been cultivated as a healthy berry [2]. The berry of L. caerulea L. is rich in polyphenols, particularly anthocyanins (mainly cyanidin 3-glucoside (C3G) [3]), and possesses multiple biological functions [4]. In our previous studies, L. caerulea L. berry polyphenols (LCBP) have been shown to be able to protect mice against high fat diet-induced non-alcoholic fatty liver disease (NAFLD), by enhancing the antioxidant capability and inhibiting the production of inflammatory cytokines of mice $[5,6]$. Recent 
studies have suggested that endotoxins or alcohols produced by gut microbiota potentially promote the progression of NAFLD $[7,8]$, and polyphenols may exert biological functions through interaction with gut microbiota [9]. Thus, we hypothesized that polyphenols from Lonicera caerulea L. berries attenuate NAFLD by regulating the intestinal bacterial community.

As a common liver disease, NAFLD has been a non-negligible threat to human health worldwide, with prevalence estimates ranging from $25 \%$ to $45 \%$ in most studies [10]. Although pathogenesis of NAFLD is complicated, factors such as high energy intake, insulin resistance, and metabolic syndrome are reported to be closely related to it [11]. Normal weight people may also develop NAFLD, although obese people have a higher risk. The presence of hepatic inflammation is considered as the most important determinant of outcome, because it promotes fibrosis in liver and leads NAFLD to the progressive non-alcoholic steatohepatitis (NASH), which can increase the risk of cirrhosis [10]. Therefore, inflammatory cytokines, triggered by endotoxins/lipopolysaccharides of intestinal bacteria $[7,12]$, are considered to be important mediators and potential biomarkers in the pathogenesis of NAFLD [13].

Based on the information about the potential pathogenesis of NAFLD, and our previous results concerning the functions of LCBP, the present study is designed to clarify the regulatory effects of LCBP on inflammatory cytokines, endotoxin production, and correlated gut microbiota in a mouse model.

\section{Results}

\subsection{Regulatory Effects of LCBP on Inflammatory Cytokines}

Our previous study showed that LCBP improved hepatic steatosis and insulin resistance [6], which is supposed to be mediated by cytokines [14]. Thus, a total number of 23 kinds of cytokines in mouse serum were simultaneously measured by multiplex technology, to understand the effects of LCBP on the cytokine network in this study. As shown in Figure 1, serum levels of IL-2 (A), IL-6 (B), MCP-1 (C), and TNF- $\alpha$ (D) were significantly increased $(P<0.05)$ in high fat diet (HFD)-fed mice, compared with normal diet (ND)-fed mice, while the amounts of other cytokines were not changed (data not shown). Supplementation with $0.5-1 \%$ of LCBP decreased $(P<0.05)$ the four cytokines to normal control level, especially serum levels of IL-2 (Figure 1A) and TNF- $\alpha$ (Figure 1D) in mice fed with a HFD containing $1 \%$ of LCBP, which were even lower than $(P<0.05)$ that in ND-fed mice. However, there was no significance between serum levels of cytokines in mice fed with ND or ND containing $1 \%$ of LCBP.

\subsection{LCBP Decreased Endotoxin Level in Bboth Serum and Liver}

As endotoxins from gut bacteria might be the trigger of inflammation in NAFLD [7], we next measured the levels of endotoxins in serum and liver homogenates of mice. As shown in Figure 2, a HFD increased significantly endotoxin levels in both serum and liver, whereas the levels of endotoxins were decreased $(p<0.05)$ by supplementing with $0.5-1 \%$ of LCBP. No significant difference was observed between the ND and ND $+1 \%$ LCBP groups. 
(A)

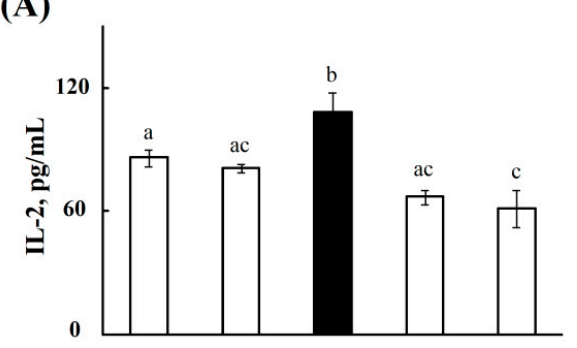

(C)

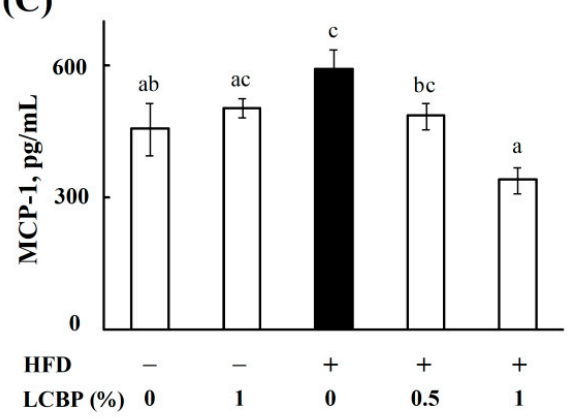

(B)

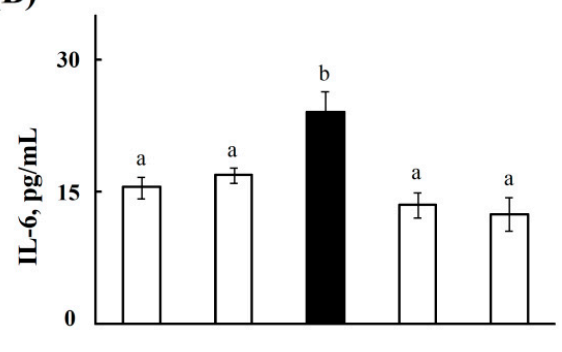

(D)

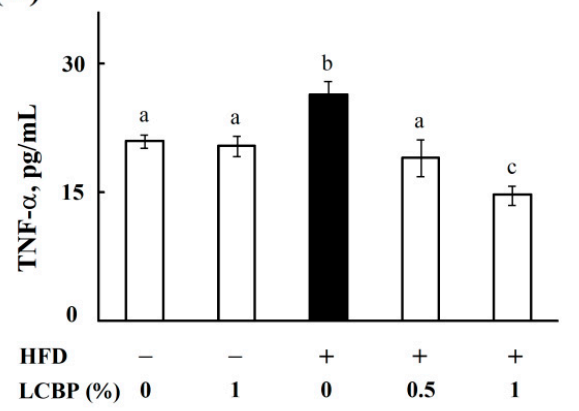

Figure 1. The effect of LCBP on serum levels of cytokine. IL-2 (A), IL-6 (B), MCP-1 (C), and TNF- $\alpha$ (D) were measured by using a Bio-Plex Pro Mouse Cytokine 23-Plex Panel kit (Bio-Rad Laboratories, Inc., Hercules, CA, USA). The data represent mean $\pm \mathrm{SD}$ of four mice. Bars with different letters differ significantly $(p<0.05)$. HFD, High fat diet; LCBP, Lonicera caerulea L. berry polyphenols; IL, interleukin; MCP-1, monocytes chemotactic protein-1; TNF- $\alpha$, tumor necrosis factor-alpha.

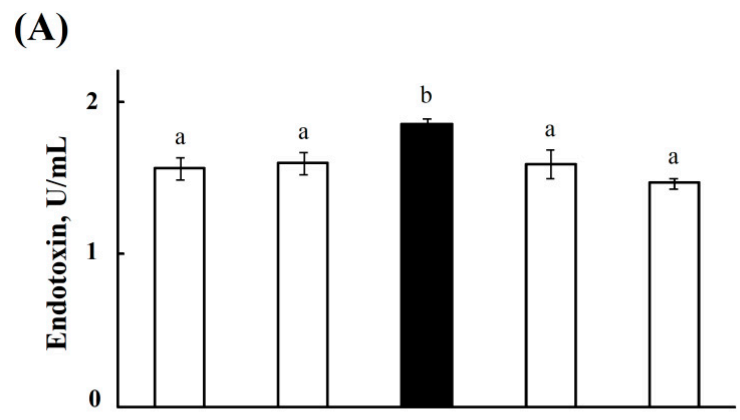

(B)

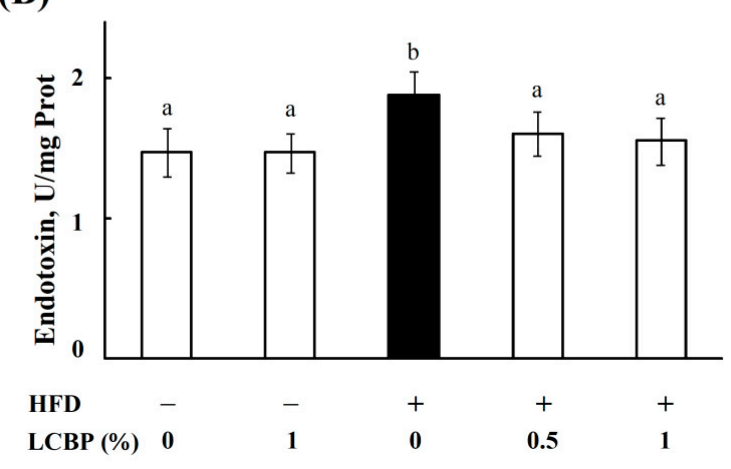

Figure 2. The effect of LCBP on endotoxin levels in serum and liver. The level of endotoxin in mouse serum (A) and liver (B) was measured by using a Pierce LAL Chromogenic Endotoxin Quantitation Kit (Thermo Fisher Scientific Inc., Rockford, IL, USA). The data represents the mean \pm SD of four mice. Bars with different letters differ significantly $(p<0.05)$. HFD, high fat diet; LCBP, Lonicera caerulea L. berry polyphenols. 


\subsection{Modulation of Gut Microbiota by LCBP}

To further understand the effects of LCBP on gut bacteria, the composition and relative abundance of microbiota were determined by using high throughput 16 rRNA gene sequencing. The diversity of fecal microbiota is shown in Table 1, HFD increased observed species richness, and Shannon diversity, compared with the ND group. Supplementation with LCBP in a HFD decreased the diversity of fecal microbiota, whereas supplementation with a ND increased the diversity.

Table 1. The effect of LCBP on the diversity of gut microbiota.

\begin{tabular}{ccccc}
\hline Description & PD_whole_tree & Chao1 & Observed_species & Shannon \\
\hline ND & 21.62 & 812.70 & 381.30 & 4.87 \\
ND + 1\% LCBP & 25.58 & 1135.23 & 515.60 & 5.75 \\
HFD & 24.84 & 1377.65 & 486.00 & 5.38 \\
HFD + 0.5\% LCBP & 22.79 & 1216.37 & 466.90 & 5.11 \\
HFD + 1\% LCBP & 21.17 & 1015.80 & 398.00 & 5.01 \\
\hline
\end{tabular}

Notes. Phylogenetic diversity (PD)_whole_tree, Chao1, and Observed species are species richness indices, and Shannon index reflects the diversity of gut microbiota. ND, normal diet group; HFD, high fat diet group; LCBP, Lonicera caerulea L. berry polyphenols.

The relative abundance of bacteria at the phylum level showed that, compared with the ND group, the abundance of Firmicutes was increased in the HFD group, while the abundance of Proteobacteria, Deferribacteres, and Actinobacteria were decreased (Figure 3A). However, supplementation with LCBP dose-dependently decreased the abundance of Firmicutes and increased the abundance of Bacteroidetes, while the abundance of Proteobacteria was also increased. Verrucomicrobia (mostly Akkermansia, as shown in Figure S2) was increased only in the HFD + 1\% LCBP group. The ratio of Firmicutes to Bacteroidetes was increased in the HFD group, but dose-dependently decreased by supplementing with $0.5-1 \%$ of LCBP (Figure 3B).

Further analysis at genus level revealed that supplementation with LCBP increased the relative abundance of genera belonging to the phylum of Bacteroidetes (Figure 4), including Bacteroides, belonging to the family of Bacteroidaceae, order of Bacteroidales, class of Bacteroidia; and Parabacteroides, belonging to the family of Porphyromonadaceae, order of Bacteroidales, class of Bacteroidia, as well as another two undefined genera, belonging to the order of Bacteroidales, family of Rikenellaceae, order of Bacteroidales, class of Bacteroidia.

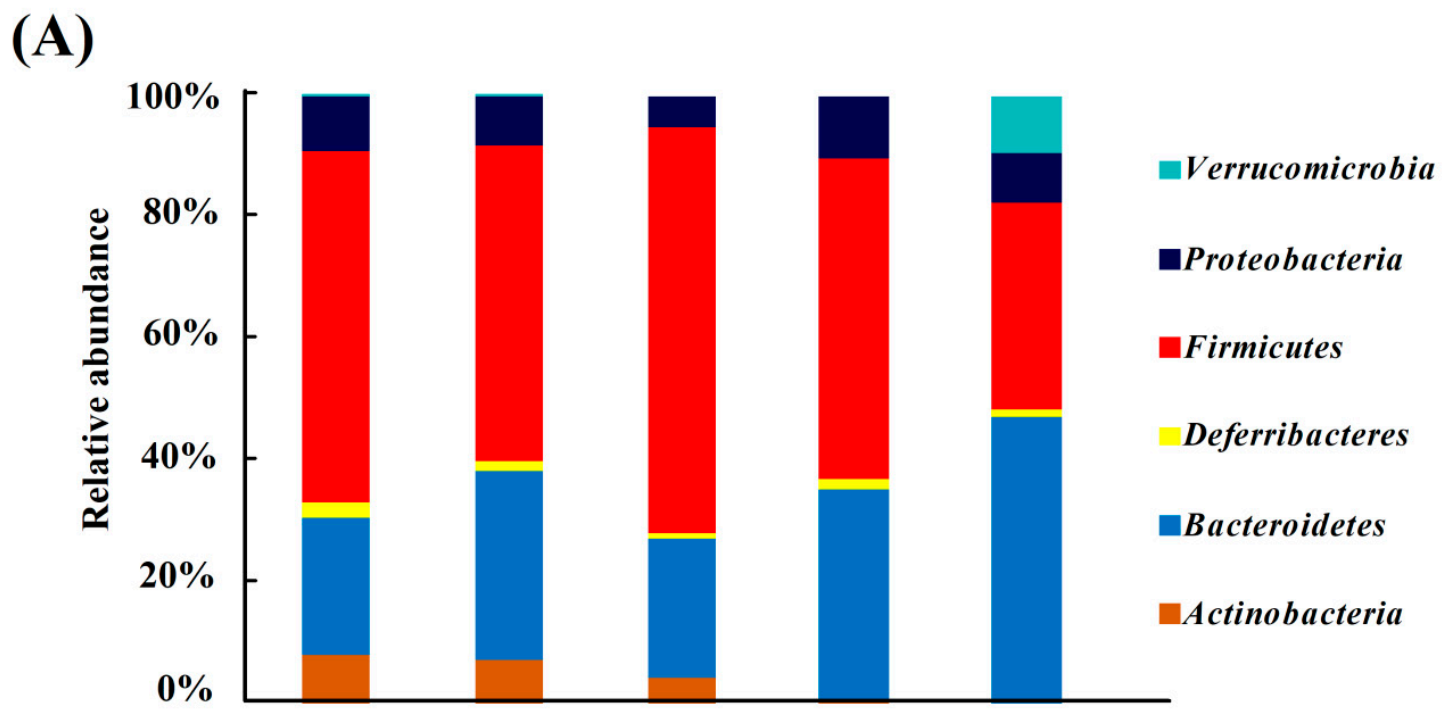

Figure 3. Cont. 
(B)

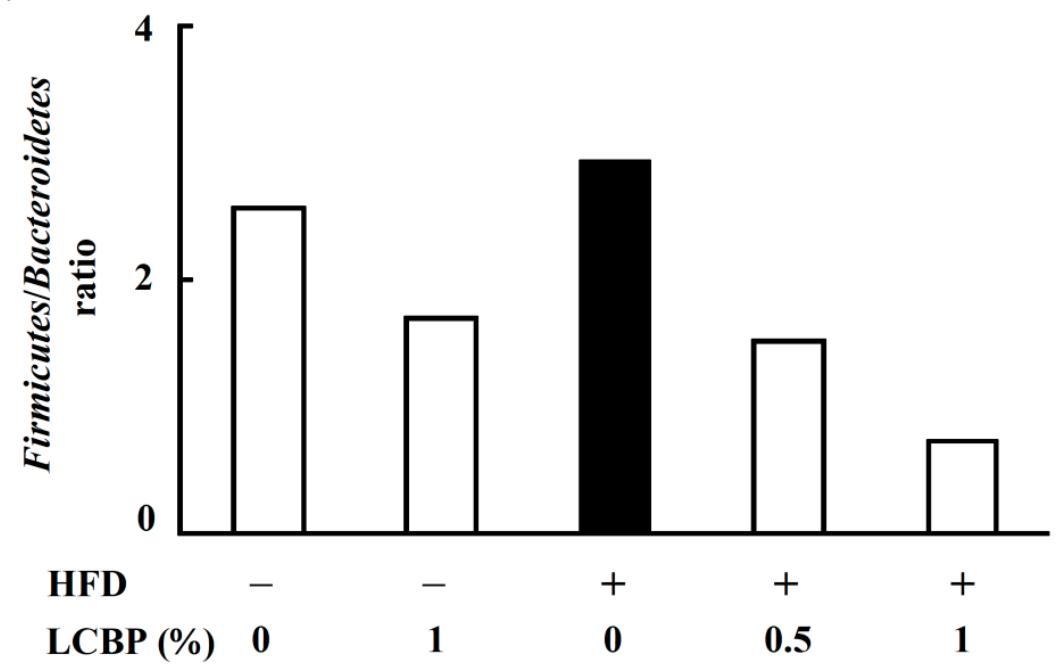

Figure 3. Modulation of gut microbiota by LCBP at the phylum level. Fecal microbiota were characterized by $16 \mathrm{~S}$ rRNA gene sequencing. (A) The relative abundance of bacteria including Verrucomicrobia, Proteobacteria, Firmicutes, Deferribacteres, Bacteroidetes and Actinobacteria, at the phylum level. (B) The ratio of Firmicutes to Bacteroidetes, based on their relative abundance. HFD, high fat diet; LCBP, Lonicera caerulea L. berry polyphenols.
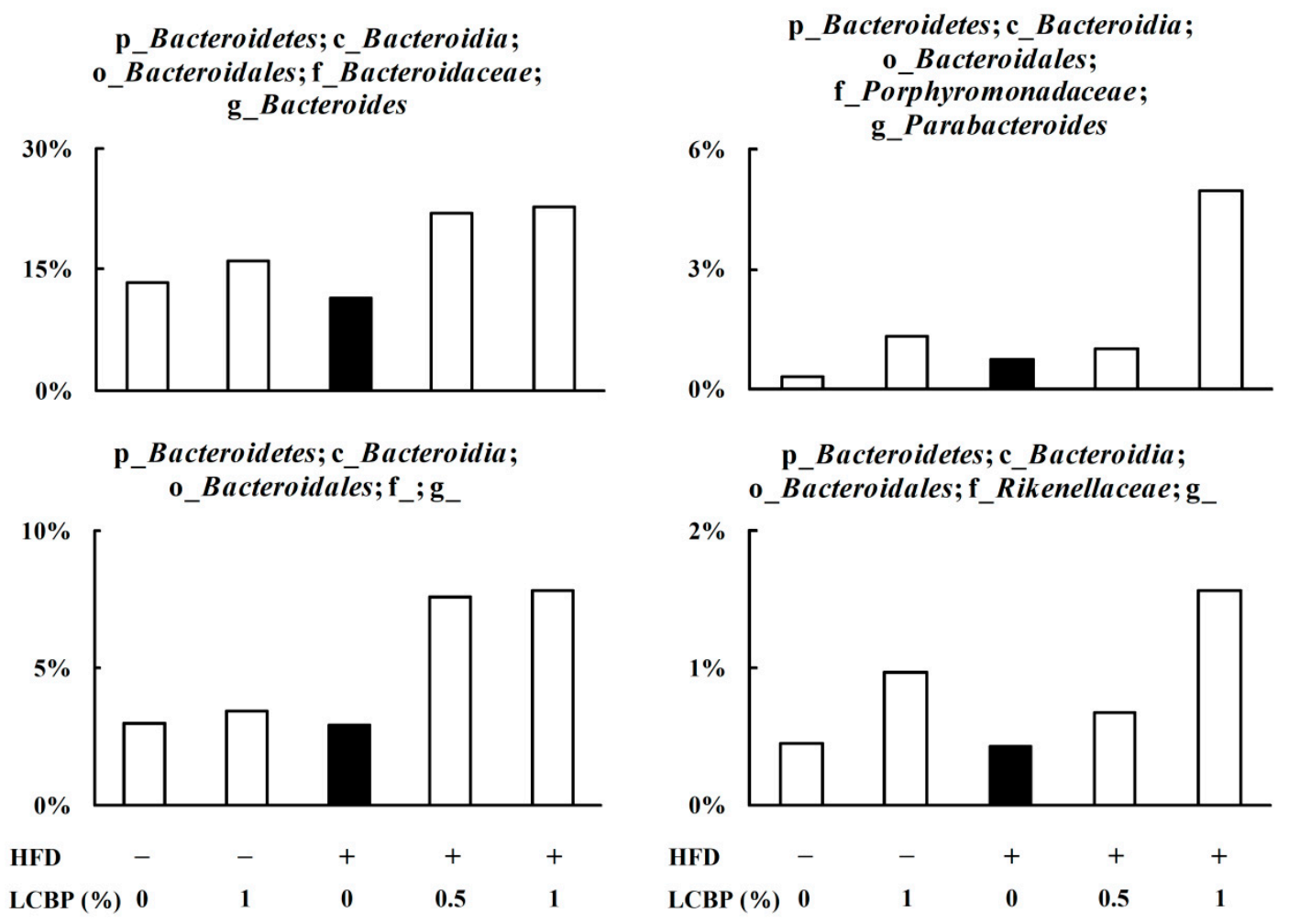

Figure 4. The effect of LCBP on the bacterial genera belonging to Bacteroidetes. Fecal microbiota were characterized by $16 \mathrm{~S}$ rRNA gene sequencing, and the data represents the relative abundance of each bacterial genus. $p_{-}, c_{-}, o_{-}, f_{-}$, or $g_{-}$represents phylum, class, order, family, and genus, respectively, and a blank after the letter means undefined. HFD, high fat diet; LCBP, Lonicera caerulea $\mathrm{L}$. berry polyphenols. 
However, LCBP decreased the relative abundance of the p_Firmicutes (Figure 5), including Staphylococcus, belonging to the family of Staphylococcaceae, order of Bacillale, class of Bacilli; Lactobacillus, belonging to the family of Lactobacillacea, order of Lactobacillales, class of Bacilli; Oscillospira, belonging to the family of Ruminococcaceae, order of Clostridiales, class of Clostridia; Ruminococcus, belonging to the family of Lachnospiraceae, order of Clostridiales, class of Clostridia; and another two undefined genera belonging to the family of Clostridiaceae, order of Clostridiales, class of Clostridia; and family of Ruminococcaceae, order of Clostridiales, class of Clostridia.

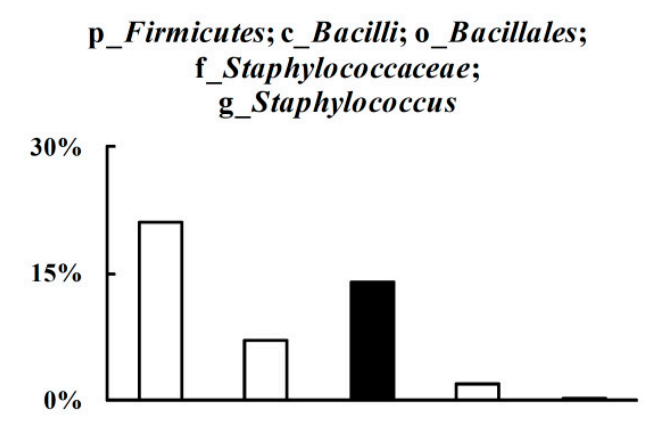

p_Firmicutes; c_Clostridia;

o_Clostridiales; f_Ruminococcaceae; g_Oscillospira

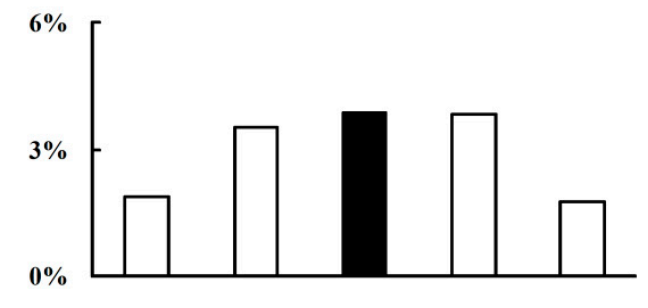

p_Firmicutes; $\mathbf{c} \_$Clostridia ; o_Clostridiales; f_Clostridiaceae; $\mathrm{g}_{\text {_ }}$

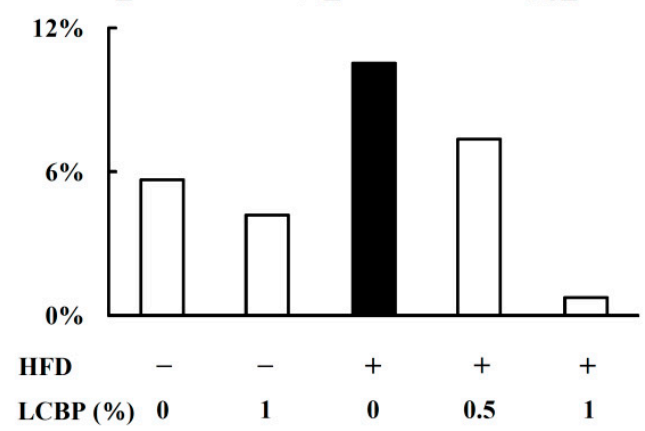

p_Firmicutes; c_Bacilli; o_Lactobacillales; $\mathbf{f}$ _Lactobacillaceae; g_Lactobacillus

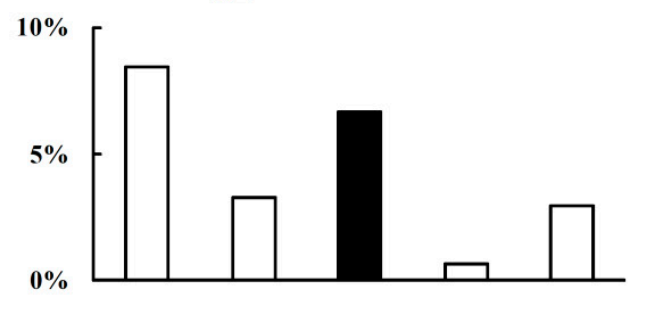

p_Firmicutes; c_Clostridia ; o_Clostridiales; $\mathrm{f} \_$Lachnospiraceae; g_Ruminococcus

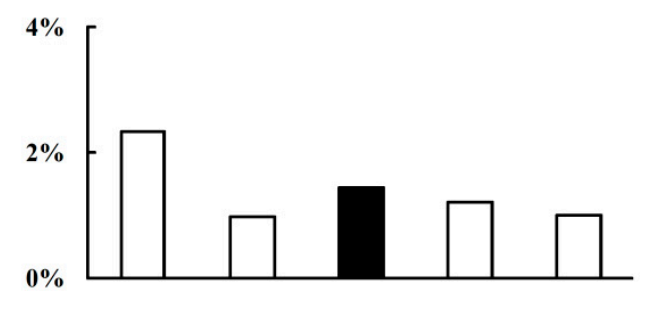

p_Firmicutes; c_Clostridia ; o_Clostridiales; $\mathbf{f} \_$Rüminococcaceae; g_

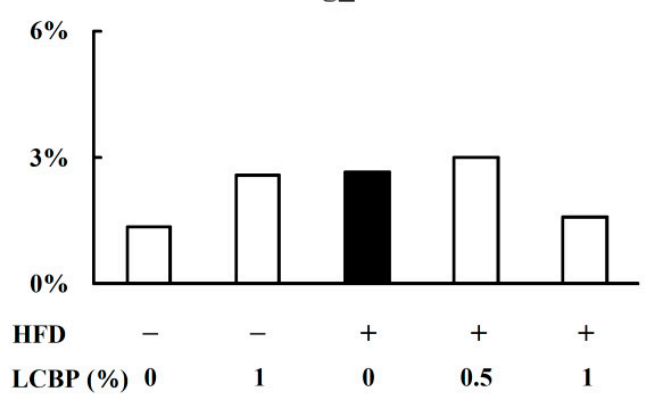

Figure 5. The effect of LCBP on the relative abundance of bacterial genera belonging to Firmicutes. Fecal microbiota were characterized by $16 \mathrm{~S}$ rRNA gene sequencing, and the data represents the relative abundance of each bacterial genus. $\mathrm{p}_{-}, \mathrm{c}_{-}, \mathrm{o}_{-}, \mathrm{f}_{-}$or $\mathrm{g}_{-}$represents phylum, class, order, family, and genus, respectively, and a blank after the letter means undefined. HFD, High fat diet; LCBP, Lonicera caerulea L. berry polyphenols.

\section{Discussion}

The gut microbiota have drawn much attention in metabolic diseases recently, since gut dysbiosis has close ties to the metabolic syndrome [15], obesity [16], diabetes [17], and cardiovascular diseases [18]. Animal studies have suggested that gut microbiota might be an important player in the pathogenesis of NAFLD [19]. Characterization of fecal microbiota in NAFLD patients also revealed that the severity of NAFLD associates with gut dysbiosis and a shift in the metabolic function of the gut microbiota [20]. Gut-derived inflammation may play an important role in the onset and progression of 
NAFLD, and actually around 40\% of inflammatory bowel disease patients also suffer from NAFLD [21]. The dysbiosis of gut microbiota may damage the intestinal epithelial barrier, and increase the intestinal permeability, which gives a way for bacteria and endotoxins to penetrate through the gut-vascular barrier, and thus promotes systemic and hepatic inflammation [22]. In the present study, HFD caused an increase in the level of endotoxins in both serum and liver of mouse, and enhanced serum levels of inflammatory cytokines including IL-2, IL-6, TNF- $\alpha$, and MCP-1, which suggesting that a HFD potentially caused the dysbiosis of gut microbiota, and IL-2, IL-6, TNF- $\alpha$, and MCP-1 were the potential primary response inflammatory cytokines in NAFLD. Supplementation of LCBP in the diet reduced endotoxin levels, with decreased levels of cytokines. The results indicated that LCBP might affect gut bacteria to re-establish the micro-ecological balance, as other studies also suggested, polyphenols potentially exert function through their interaction with gut microbiota [9].

Multiple species of bacteria have been reported to be closely associated with NAFLD. Characterization of gut microbiota in human stool samples revealed that a significant decrease in Bacteroidetes at the phylum level was found in NAFLD patients, compared with healthy controls [7,23], while the relative abundance of Actinobacteria [23] was increased. A recent study revealed that obese people have a higher Firmicutes / Bacteroidetes ratio, compared to normal weight people in the adult Ukrainian population [24]. In this study, the HFD increased the abundance of Firmicutes, but Bacteroidetes did not change. Supplementation of LCBP in a normal diet (ND) or HFD dose-dependently increased the relative abundance of Bacteroidetes, and decreased the abundance of Firmicutes, and, in this way, LCBP decreased the Firmicutes / Bacteroidetes ratio in a dose-dependent manner. At the genus level, Bacteroides and Parabacteroides were identified as the up-regulated bacterias by LCBP, while other two bacterial genera belong to the order of Bacteroidales and family of Rikenellaceae were also increased. Previous studies demonstrated that the relative abundance of Bacteroides and Parabacteroides were decreased in the gut of NAFLD patients [23], or HFD-induced rats [25]. However, the abundance of Bacteroides and Parabacteroides between ND and HFD groups had little change in this study. On the other hand, six genera belong to the phylum of Firmicutes, including Staphylococcus, Lactobacillus, Ruminococcus, and Oscillospira were decreased by LCBP. Recent studies suggested that the abundance of Lactobacillus, Ruminococcus, and Oscillospira were increased with age and associated with intestinal permeability, systemic inflammation, and macrophage dysfunction [26,27]. This may explain the increase in the levels of endotoxin and inflammatory cytokines in circulation. It is also noteworthy that the abundance of Akkermansia, bacteria that can prevent the development of obesity and associated complications $[28,29]$, was increased in the HFD + 1\% LCBP group, although rarely found in other groups. This will be further studied in future works.

In conclusion, supplementation with $0.5-1 \%$ of LCBP in diet decreased significantly the levels of inflammatory cytokines, including IL-2, IL-6, MCP-1, and TNF- $\alpha$, in serum, as well as endotoxin levels in both serum and liver in a mice model of NAFLD. Fecal microbiota characterization by high throughput $16 \mathrm{~S}$ rRNA gene sequencing demonstrated that LCBP reduced the Firmicutes / Bacteroidetes ratio, which was increased by a HFD. These data provided a scientific basis for understanding the pathogenesis of NAFLD, and the preventive effects of NAFLD by intervention of polyphenols from the Lonicera caerulea L. berry.

\section{Materials and Methods}

\subsection{Chemicals and Reagents}

Choline chloride, lard oil, and methionine were purchased from Sigma-Aldrich (Tokyo, Japan). Casein, cellulose, corn starch, mineral mix, sucrose, and vitamin mix were purchased from Oriental Yeast Co., Ltd., (Tokyo, Japan). LCBP were extracted as described previously [3]. Briefly, the Lonicera caerulea L. berries, from the Jilin province of China, were homogenized in $75 \%$ aqueous ethanol (250 g/L) for $60 \mathrm{~min}$, and then filtered under reduced pressure. The filtrates were purified on a column packed with nonionic polystyrene-divinylbenzene resin (D101, Shanghai Mosu Science 
Equipment Co., Ltd, Shanghai, China), and then freeze-dried into powder. The retention profiles of HPLC analysis showed that C3G (59.5\%) and -(-)epicatechin (EC) (25.5\%) were the major phenolic components at $280 \mathrm{~nm}$, and other minor anthocyanins, including cyanidin 3-rutinoside (1.8\%), cyanidin 3,5-diglucoside (1.3\%), peonidin 3-glucoside (7.2\%), peonidin 3-rutinoside (1.9\%), and pelargonidin 3 -glucoside $(2.3 \%)$ were also detected at $520 \mathrm{~nm}$. The quantitative analysis by HPLC (Figure S1), using standards, indicated that each milligram of LCBP contains $0.37 \mathrm{mg}$ C3G and $0.23 \mathrm{mg}$ EC.

\subsection{Mouse Model of NAFLD}

The animal experiment was conducted according to the guidelines of the Animal Care and Use Committee of Kagoshima University (Permission No. A12005), Japan. Twenty C57BL/6N mice (male, 5 weeks of age) were purchased from SLC Inc. (Shizuoka, Japan), and housed separately in cages with wood shaving bedding under controlled temperature $\left(25^{\circ} \mathrm{C}\right)$ and light $(12 \mathrm{~h}$ light/day), and had free access to feed and water. After being accommodated for 1 week, the mice were randomly divided into 5 groups $(n=4)$ : A normal diet (ND) group, a ND + 1\% LCBP group, a high fat diet (HFD) group, a HFD $+0.5 \%$ LCBP group, and a HFD $+1 \%$ LCBP group. Mice in different groups were fed the corresponding diets (as described in Table S1) for 45 days.

\subsection{Measurement of Cytokines}

Blood sera of mice were obtained by centrifuging at $1500 \times g$ for $10 \mathrm{~min}$ after coagulation at the end of the experiment, and serum levels of cytokines were measured by multiplex technology, as described previously [30]. In brief, cytokines, including interleukin (IL)-1 $\alpha$, IL-1 $\beta$, IL-2, IL-3, IL-4, IL-5, IL-6, IL-9, IL-10, IL-12(p40), IL-12(p70), IL-13, IL-17, eotaxin, granulocyte colony-stimulating factor (G-CSF), granulocyte-macrophage colony-stimulating factor (GM-CSF), interferon-gamma (IFN- $\gamma$ ), keratinocyte-derived cytokine (KC), monocyte chemotactic protein-1 (MCP-1), macrophage inflammatory protein (MIP)-1 $\alpha$, MIP-1 $\beta$, RANTES, and tumor necrosis factor-alpha (TNF- $\alpha$ ), were measured with a Bio-Plex Pro Mouse Cytokine 23-Plex Panel kit (Bio-Rad Hercules, Hercules, CA, USA) by using a Bio-Plex 200 System (Bio-Rad Hercules, Hercules, CA, USA) according to the manufacturer's instruction.

\subsection{Measurement of Endotoxins}

To measure the levels of toxins in the liver, equal amounts of liver tissues were homogenized in normal saline $(0.1 \mathrm{~g} / \mathrm{mL})$ with a Speed-Mill PLUS homogenizer (Analytik Jena, Jena, Germany), and the supernatants were obtained by being centrifuged at $3500 \times g$ for $10 \mathrm{~min}$. The levels of endotoxins in mouse serum and liver homogenates were measured by using a Pierce LAL Chromogenic Endotoxin Quantitation Kit (Thermo Fisher Scientific Inc., Rockford, IL, USA), according to the manufacturer's manual.

\subsection{Characterization of Gut Microbiota by $16 S$ rRNA Gene Sequencing}

Feces samples of each mouse were collected for analyzing the composition of gut bacterial communities, at the end of experiment. Briefly, the feces of each mouse were collected separately into a $1.5 \mathrm{~mL}$ tube, and freeze-dried to achieve constant weight. An equal amount of feces from each group was washed by DNase-free water to clean the surface, and then fecal DNA was extracted using a QIAamp PowerFecal DNA Kit (QIAGEN, Venlo, Netherlands), following the manufacturer's manual. The V3-V4 region of the $16 \mathrm{~S}$ rRNA gene was amplified using forward primer $341 \mathrm{~F}$ (5'-ACACTCTTTCCCTACACGACGCTCTTCCGATCT-NNNNN-CCTACGGGNGGCWGCAG-3') and reverse primer $805 \mathrm{R}\left(5^{\prime}\right.$-GTGACTGGAGTTCA GACGTGTGCTCTTCCGATCT-NNNNN-GACTACHV GGGTATCTAATCC $\left.-3^{\prime}\right)$. PCR cycling conditions consisted of an initial denaturation of 2 min at $94{ }^{\circ} \mathrm{C}$, and 25 cycles of $30 \mathrm{~s}$ at $94{ }^{\circ} \mathrm{C}, 30 \mathrm{~s}$ at $55{ }^{\circ} \mathrm{C}$, and $30 \mathrm{~s}$ at $72{ }^{\circ} \mathrm{C}$. The quality of the amplified $16 \mathrm{~S}$ rRNA gene was checked on $0.8 \%$ agarose gels. The PCR products were amplified in a second PCR employing index primer (F: 5'-AATGATACGGCGACCACCGAGATCTACAC- 
Index2-ACACTCTTTCCCTACACGACGC-3'; R: 5'-CAAGCAGAAGACGGCATACGAGAT-Index1-GT GACTGGAGTTCAGACGTGTG-3'). This PCR was run for 2 min at $94{ }^{\circ} \mathrm{C}$, followed by 10 cycles of $30 \mathrm{~s}$ at $94{ }^{\circ} \mathrm{C}, 30 \mathrm{~s}$ at $60^{\circ} \mathrm{C}$, and $30 \mathrm{~s}$ at $72{ }^{\circ} \mathrm{C}$. Amplicon sequencing was performed on the Illumina MiSeq System at Bioengineering Lab. Co., Ltd. (Atsugi, Kanagawa, Japan).

\subsection{Statistical Analysis}

Results are expressed as means \pm SD. Significant differences between groups were determined using one-way analysis of variance (ANOVA) tests, followed by Duncan's Multiple Range test (SPSS19, IBM Corp., Armonk, NY, USA). A probability of $p<0.05$ was considered significant.

Supplementary Materials: The Supplementary Materials are available online.

Author Contributions: Conceptualization, S.W. and D.-X.H.; Formal Analysis, R.H. and M.L.; Investigation, S.W., H.N., and K.R.; Resources, J.H., X.H., and H.Z.; Writing-Original Draft Preparation, S.W.; Writing-Review and Editing, D.-X.H. and J.H.; Project Administration, S.W. and D.-X.H.

Funding: This work was funded by National Natural Science Foundation of China (No. 31741115).

Acknowledgments: We appreciate the support from the Hundred-Talent Program of the Hunan province, and the State Key Laboratory of Animal Nutrition.

Conflicts of Interest: The authors declare no conflict of interest.

\section{References}

1. Rupasinghe, H.P.; Boehm, M.M.; Sekhon-Loodu, S.; Parmar, I.; Bors, B.; Jamieson, A.R. Anti-Inflammatory Activity of Haskap Cultivars is Polyphenols-Dependent. Biomolecules 2015, 5, 1079-1098. [CrossRef] [PubMed]

2. Oszmiański, J.; Wojdyło, A.; Lachowicz, S. Effect of dried powder preparation process on polyphenolic content and antioxidant activity of blue honeysuckle berries (Lonicera caerulea L. var kamtschatica). LWT Food Sci. Technol. 2016, 67, 214-222. [CrossRef]

3. Wu, S.; He, X.; Wu, X.; Qin, S.; He, J.; Zhang, S.; Hou, D.-X. Inhibitory effects of blue honeysuckle (Lonicera caerulea L.) on adjuvant-induced arthritis in rats: Crosstalk of anti-inflammatory and antioxidant effects. J. Funct. Foods 2015, 17, 514-523. [CrossRef]

4. Becker, R.; Szakiel, A. Phytochemical characteristics and potential therapeutic properties of blue honeysuckle Lonicera caerulea L. (Caprifoliaceae). J. Herb. Med. 2018. [CrossRef]

5. Wu, S.; Yano, S.; Hisanaga, A.; He, X.; He, J.; Sakao, K.; Hou, D.X. Polyphenols from Lonicera caerulea L. berry attenuate experimental nonalcoholic steatohepatitis by inhibiting proinflammatory cytokines productions and lipid peroxidation. Mol. Nutr. Food Res. 2017, 61. [CrossRef]

6. Liu, M.; Tan, J.; He, Z.; He, X.; Hou, D.-X.; He, J.; Wu, S. Inhibitory effect of blue honeysuckle extract on high-fat-diet-induced fatty liver in mice. Anim. Nutr. 2018, 4, 288-293. [CrossRef] [PubMed]

7. Kessoku, T.; Imajo, K.; Honda, Y.; Kato, T.; Ogawa, Y.; Tomeno, W.; Higurashi, T.; Yoneda, M.; Shimakawa, M.; Tanaka, Y.; et al. Characteristics of Fecal Microbiota in Japanese Patients with Nonalcoholic Fatty Liver Disease: A Connection among Gut-Permeability, Endotoxin and NAFLD. Gastroenterol. 2017, 152, S1200. [CrossRef]

8. Zhu, L.; Baker, R.D.; Zhu, R.; Baker, S.S. Gut microbiota produce alcohol and contribute to NAFLD. Gut 2016, 65, 1232. [CrossRef] [PubMed]

9. Tomas-Barberan, F.A.; Selma, M.V.; Espin, J.C. Polyphenols' Gut Microbiota Metabolites: Bioactives or Biomarkers? J. Agric. Food Chem. 2018, 66, 3593-3594. [CrossRef] [PubMed]

10. Rinella, M.E. Nonalcoholic fatty liver disease: A systematic review. JAMA 2015, 313, 2263-2273. [CrossRef] [PubMed]

11. Younossi, Z.; Anstee, Q.M.; Marietti, M.; Hardy, T.; Henry, L.; Eslam, M.; George, J.; Bugianesi, E. Global burden of NAFLD and NASH: Trends, predictions, risk factors and prevention. Nat. Rev. Gastroenterol. Hepatol. 2018, 15, 11-20. [CrossRef] [PubMed]

12. Kitabatake, H.; Tanaka, N.; Fujimori, N.; Komatsu, M.; Okubo, A.; Kakegawa, K.; Kimura, T.; Sugiura, A.; Yamazaki, T.; Shibata, S.; et al. Association between endotoxemia and histological features of nonalcoholic fatty liver disease. World J. Gastroenterol. 2017, 23, 712-722. [CrossRef] [PubMed] 
13. Stojsavljevic, S.; Gomercic Palcic, M.; Virovic Jukic, L.; Smircic Duvnjak, L.; Duvnjak, M. Adipokines and proinflammatory cytokines, the key mediators in the pathogenesis of nonalcoholic fatty liver disease. World J. Gastroenterol. 2014, 20, 18070-18091. [CrossRef] [PubMed]

14. Ueki, K.; Kondo, T.; Tseng, Y.H.; Kahn, C.R. Central role of suppressors of cytokine signaling proteins in hepatic steatosis, insulin resistance, and the metabolic syndrome in the mouse. Proc. Natl. Acad. Sci. USA 2004, 101, 10422-10427. [CrossRef] [PubMed]

15. Vrieze, A.; Van Nood, E.; Holleman, F.; Salojarvi, J.; Kootte, R.S.; Bartelsman, J.F.; Dallinga-Thie, G.M.; Ackermans, M.T.; Serlie, M.J.; Oozeer, R.; et al. Transfer of intestinal microbiota from lean donors increases insulin sensitivity in individuals with metabolic syndrome. Gastroenterol. 2012, 143. [CrossRef] [PubMed]

16. Turnbaugh, P.J.; Hamady, M.; Yatsunenko, T.; Cantarel, B.L.; Duncan, A.; Ley, R.E.; Sogin, M.L.; Jones, W.J.; Roe, B.A.; Affourtit, J.P.; et al. A core gut microbiome in obese and lean twins. Nature 2009, 457, 480-484. [CrossRef] [PubMed]

17. Qin, J.; Li, Y.; Cai, Z.; Li, S.; Zhu, J.; Zhang, F.; Liang, S.; Zhang, W.; Guan, Y.; Shen, D.; et al. A metagenomewide association study of gut microbiota in type 2 diabetes. Nature 2012, 490, 55-60. [CrossRef]

18. He, Y.; Wu, W.; Zheng, H.M.; Li, P.; McDonald, D.; Sheng, H.F.; Chen, M.X.; Chen, Z.H.; Ji, G.Y.; Zheng, Z.D.; et al. Regional variation limits applications of healthy gut microbiome reference ranges and disease models. Nat. Med. 2018, 24, 1532-1535. [CrossRef]

19. Boursier, J.; Diehl, A.M. Implication of gut microbiota in nonalcoholic fatty liver disease. PLoS Pathog. 2015, 11. [CrossRef]

20. Boursier, J.; Mueller, O.; Barret, M.; Machado, M.; Fizanne, L.; Araujo-Perez, F.; Guy, C.D.; Seed, P.C.; Rawls, J.F.; David, L.A.; et al. The severity of nonalcoholic fatty liver disease is associated with gut dysbiosis and shift in the metabolic function of the gut microbiota. Hepatology 2016, 63, 764-775. [CrossRef]

21. Sartini, A.; Gitto, S.; Bianchini, M.; Verga, MC.; Di Girolamo, M.; Bertani, A.; Del Buono, M.; Schepis, F.; Lei, B.; De Maria, N.; et al. Non-alcoholic fatty liver disease phenotypes in patients with inflammatory bowel disease. Cell Death Dis. 2018, 9. [CrossRef] [PubMed]

22. Cheng, C.; Tan, J.; Qian, W.; Zhang, L.; Hou, X. Gut inflammation exacerbates hepatic injury in the high-fat diet induced NAFLD mouse: Attention to the gut-vascular barrier dysfunction. Life Sci. 2018, 209, 157-166. [CrossRef]

23. Del Chierico, F.; Nobili, V.; Vernocchi, P.; Russo, A.; Stefanis, C.; Gnani, D.; Furlanello, C.; Zandona, A.; Paci, P.; Capuani, G.; et al. Gut microbiota profiling of pediatric nonalcoholic fatty liver disease and obese patients unveiled by an integrated meta-omics-based approach. Hepatology 2017, 65, 451-464. [CrossRef] [PubMed]

24. Koliada, A.; Syzenko, G.; Moseiko, V.; Budovska, L.; Puchkov, K.; Perederiy, V.; Gavalko, Y.; Dorofeyev, A.; Romanenko, M.; Tkach, S.; et al. Association between body mass index and Firmicutes/Bacteroidetes ratio in an adult Ukrainian population. BMC Microbiol. 2017, 17. [CrossRef] [PubMed]

25. Liu, J.P.; Zou, W.L.; Chen, S.J.; Wei, H.Y.; Yin, Y.N.; Zou, Y.Y.; Lu, F.G. Effects of different diets on intestinal microbiota and nonalcoholic fatty liver disease development. World J. Gastroenterol. 2016, 22, 7353-7364. [CrossRef] [PubMed]

26. Thevaranjan, N.; Puchta, A.; Schulz, C.; Naidoo, A.; Szamosi, J.C.; Verschoor, C.P.; Loukov, D.; Schenck, L.P.; Jury, J.; Foley, K.P.; et al. Age-Associated Microbial Dysbiosis Promotes Intestinal Permeability, Systemic Inflammation, and Macrophage Dysfunction. Cell Host Microbe 2017, 21, 455-466.e4. [CrossRef] [PubMed]

27. Santisteban, M.M.; Qi, Y.; Zubcevic, J.; Kim, S.; Yang, T.; Shenoy, V.; Cole-Jeffrey, C.T.; Lobaton, G.O.; Stewart, D.C.; Rubiano, A.; et al. Hypertension-Linked Pathophysiological Alterations in the Gut. Circ. Res. 2017, 120, 312-323. [CrossRef]

28. Plovier, H.; Everard, A.; Druart, C.; Depommier, C.; Van Hul, M.; Geurts, L.; Chilloux, J.; Ottman, N.; Duparc, T.; Lichtenstein, L.; et al. A purified membrane protein from Akkermansia muciniphila or the pasteurized bacterium improves metabolism in obese and diabetic mice. Nat. Med. 2016, 23, 107-113. [CrossRef]

29. Everard, A.; Belzer, C.; Geurts, L.; Ouwerkerk, J.P.; Druart, C.; Bindels, L.B.; Guiot, Y.; Derrien, M.; Muccioli, G.G.; Delzenne, N.M.; et al. Cross-talk between Akkermansia muciniphila and intestinal epithelium controls diet-induced obesity. Proc. Natl. Acad. Sci. USA 2013, 110, 9066-9071. [CrossRef] 
30. Wu, S.; Yano, S.; Chen, J.; Hisanaga, A.; Sakao, K.; He, X.; He, J.; Hou, D.X. Polyphenols from Lonicera caerulea L. Berry Inhibit LPS-Induced Inflammation through Dual Modulation of Inflammatory and Antioxidant Mediators. J. Agric. Food Chem. 2017, 65, 5133-5141. [CrossRef]

Sample Availability: Samples of the compounds isolated from Lonicera caerulea L. berry are available from the authors.

(C) 2018 by the authors. Licensee MDPI, Basel, Switzerland. This article is an open access article distributed under the terms and conditions of the Creative Commons Attribution (CC BY) license (http://creativecommons.org/licenses/by/4.0/). 